\title{
Automatic Wandering Detection with a Microwave Doppler Sensor: Classification of Human's Presence Using Bio-Information
}

\author{
Kazuaki Shiba*, Takashi Kaburagi, Yosuke Kurihara \\ Aoyama Gakuin University, 5-10-1, Fuchinobe, Chuo-ku, Sagamihara, 252-5258, Japan \\ *Corresponding Author: c5616178@aoyama.jp
}

\begin{abstract}
The ageing population of our global society is presenting some new problems. In particular, the rate of dementia is also increasing. People with dementia (PwD) may wander outside the area controlled by the caregivers, which may result in serious accidents, such as traffic accidents. Constant monitoring is therefore required, to enable a fast response to wandering. This round-the-clock monitoring task imposes a heavy burden on caregivers, which can be reduced if reliable systems for automatically monitoring PwD in their homes can be developed. One of the fundamental features to support effective wandering detection systems is the ability to classify the presence or absence of a subject in a designated area. In this paper, we propose a novel system to classify the presence of a person within a designated area using a microwave Doppler sensor. We focus on the detection of bio-signals; in particular, of respiratory and heartbeat signals. The microwave Doppler sensor adopted in this system has two output channels, providing in-phase and quadrature signals. Four types of features are extracted from the observed signals and then input to a support vector machine algorithm, to classify the presence/absence of a subject in the target area. For the presence of a subject, two different states are considered: a rest-state and a moving-state. The rest-state corresponds to a subject quietly sitting in the designated area; the moving-state corresponds to a subject walking randomly within the designated area. The system was evaluated using 11 subjects, and exhibited a $99.73 \%$ accuracy. In particular, the absence of a subject in the area could be detected with $100 \%$ certainty. The obtained experimental results show that considering the bio-signals does, in fact, yield better results.
\end{abstract}

Keywords: nursing care, wandering, respiration, heartbeat, microwave Doppler sensor.

\section{Introduction}

The world population with 65 years or more is projected to reach 1.4 billion by $2030^{(1)}$. With this increase in age, the ratio of dementia is also increasing ${ }^{(2)}$. Therefore, the onus of nursing and caring for people with dementia $(\mathrm{PwD})$ in our global society is rising. Additionally, as has been reported ${ }^{(3)}$, the majority of PwD may at some point wander outside, which may result in serious accidents, such as traffic accidents ${ }^{(4)}$. Therefore, constant, uninterrupted monitoring is required, to enable a fast response to wandering. This round-the-clock monitoring task imposes a heavy burden on caregivers. Automatic systems capable of detecting wandering behaviors are therefore required.

Many of the existing methods for wandering detection are GPS-based ${ }^{(5,6)}$; using a mobile phone's GPS, the subject's position can be determined. However, people who may wander may not carry a mobile phone and, furthermore, GPS cannot be used indoors; this represents a severe limitation to the GPS-based approach.

One of the fundamental features of an effective wandering detection system is the ability to detect/classify the presence or absence of a subject within a designated area. Even though human detection algorithms have been previously proposed ${ }^{(7-9)}$, additional efforts can be made to improve classification accuracy. We have been developing systems that classify the presence/absence of subjects in a designated area ${ }^{(10)}$. In prior work ${ }^{(10)}$, we assumed that a subject would calmly sit in the designated area. However, to use the proposed system in real life, we need to incorporate more states in the presence classification algorithm, such as a walking state. In this work, the objective is to confirm if 
the walking state can be successfully classified as a presence.

We focus on bio-signals; in particular, on respiratory and heartbeat signals. The microwave Doppler sensor adopted in this system has two output channels, the in-phase and quadrature signals. Four types of features are calculated from the observed signals and are then input to a support vector machine (SVM) to be classified as corresponding to either a presence or an absence of the subject. The system was evaluated using 11 subjects, and exhibited a present- or absent-state classification with $99.73 \%$ accuracy.

\section{Proposed Method}

The proposed method consists of three steps: 1) Obtain the microwave Doppler sensor signals and transform them into the frequency domain. 2) Calculate the feature vector. 3) Classify the data into the present or absent classes, using an SVM. Fig. 1 shows the block diagram of the proposed system.

\subsection{Data collection and frequency analysis}

The microwave Doppler sensor used in this paper has two output signal components: i) the in-phase signal $I(t)$ and ii) the quadrature signal $Q(t)$, where $t$ denotes continuous time. Each signal is sampled, generating the $I(k)$ and $Q(k)$ sequences, respectively. $k$ denotes discrete time index. The amplitude spectra of both components $F_{I}(f)$ and $F_{Q}(f)$ are obtained using a discrete Fourier transform, where $f$ denotes discrete frequency.

\subsection{Feature vector}

In this research, we are interested in the frequency contents of human bio-signals; in particular, of respiratory and heartbeat signals. Therefore, two different bio-signals are used, to generate two types of features: a) respiration frequency and b) heartbeat frequency.

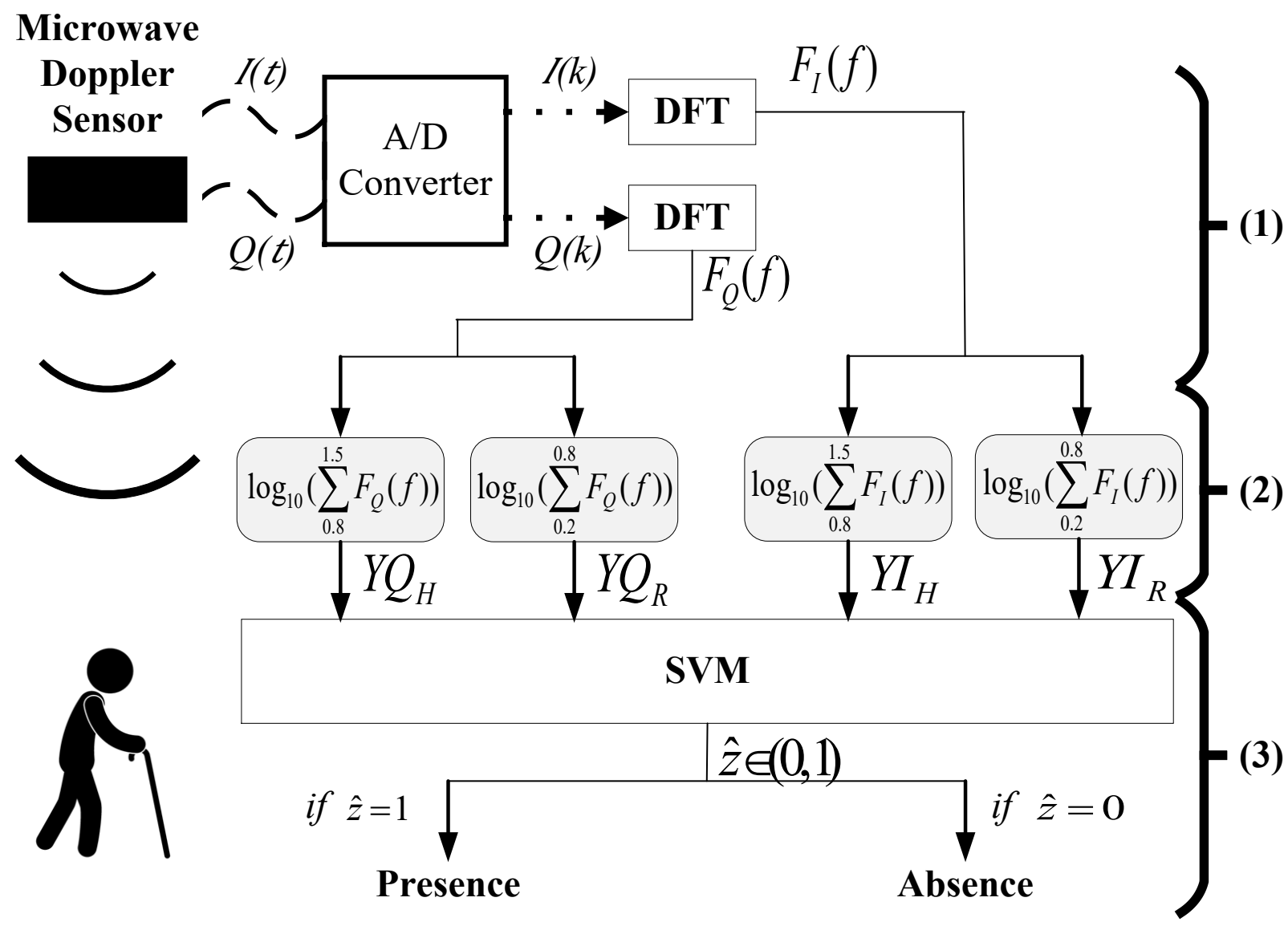

Fig.1 Overview of the proposed system. (1) Two observed signals from the microwave Doppler sensor are converted from analog to digital and demodulated to one signal. And these two signals are converted to frequency domain. (2)Then, four components of feature vector is calculated. (3) Finally, a SVM classifies a subject present or absent. 
(a) Respiration Feature Vector Components

In general, the human respiratory rate varies from 12 to 50 times per minute, which corresponds to a fundamental frequency between $0.2 \mathrm{~Hz}$ and $0.8 \mathrm{~Hz}$. Therefore, the sums of the amplitude spectrum components between $0.2 \mathrm{~Hz}$ and $0.8 \mathrm{~Hz}$ are taken as the respiration domain features $Y I_{R}$ and $Y Q_{R}$ :

$$
\begin{aligned}
& Y Q_{I}=\log _{10}\left(\sum_{0.2}^{0.8} F_{I}(f)\right) \\
& Y Q_{R}=\log _{10}\left(\sum_{0.2}^{0.8} F_{Q}(f)\right) .
\end{aligned}
$$

(b) Heartbeat Feature Vector Components

The human heartbeat rate varies between 50 and 90 beats per minute, which corresponds to a fundamental frequency between $0.8 \mathrm{~Hz}$ and $1.5 \mathrm{~Hz}$. Therefore, the sums of the amplitude spectrum components between $0.8 \mathrm{~Hz}$ and $1.5 \mathrm{~Hz}$ are taken as the heartbeat domain features $Y I_{H}$ and $Y Q_{H}$ :

$$
\begin{aligned}
& Y I_{H}=\log _{10}\left(\sum_{0.8}^{1.5} F_{I}(f)\right) \\
& Y Q_{H}=\log _{10}\left(\sum_{0.8}^{1.5} F_{Q}(f)\right) .
\end{aligned}
$$

\subsection{Present/Absent Classification}

Given the four feature vector components presented in Section $2.2\left(Y I_{R}, Y Q_{H}, Y I_{R}\right.$, and $\left.Y Q_{H}\right)$, we classify the data into the present/absent classes using an SVM. The SVM output $\hat{Z} \in\{0,1\}$ denotes the presence status of a subject within a designated area: $\hat{Z}=1$ represents the subject presence, and $\hat{Z}=0$ represents the subject absence.

\section{Experimental Setup}

We performed an experimental evaluation test to verify the accuracy of the proposed system. An overview of the experimental system is shown in Fig. 2.

\subsection{Materials}

The microwave Doppler sensor used in this paper is a IPS-154 K-band CW transceiver from InnoSenT. The in-phase and quadrature signals were amplified in the system board with a $+40 \mathrm{~dB}$ gain. An $\mathrm{A} / \mathrm{D}$ converter (AI-1608AY-USB; Contec) was connected to a Windows laptop via a USB cable. A free logging software program provided by Contec (the C-Logger) was used to sample $I(t)$ and $Q(t)$ with a $100 \mathrm{~Hz}$ sampling frequency. The collection time interval was set to 30 seconds; sequences with 3000 samples were therefore generated.

In this experiment, the target area was a $2.0 \mathrm{~m} \times 2.0 \mathrm{~m}$ square area, as shown in Fig 3. A grid of $5 \times 5=25$ positions was defined on the area, with a distance of $0.5 \mathrm{~m}$ between positions in the same grid row/column. Each position was assigned a number from 1 to 25 . The sensor device was mounted $2.3 \mathrm{~m}$ above the floor, just above the center of this experiment area (position 13). This height was selected because the celling in most residences is at approximately $2.3 \mathrm{~m}$ from the floor. Eleven subjects were considered in the experiment, after having obtained their informed consent: ten male subjects, and one female subject, ranging in age from 20 to 24 years.

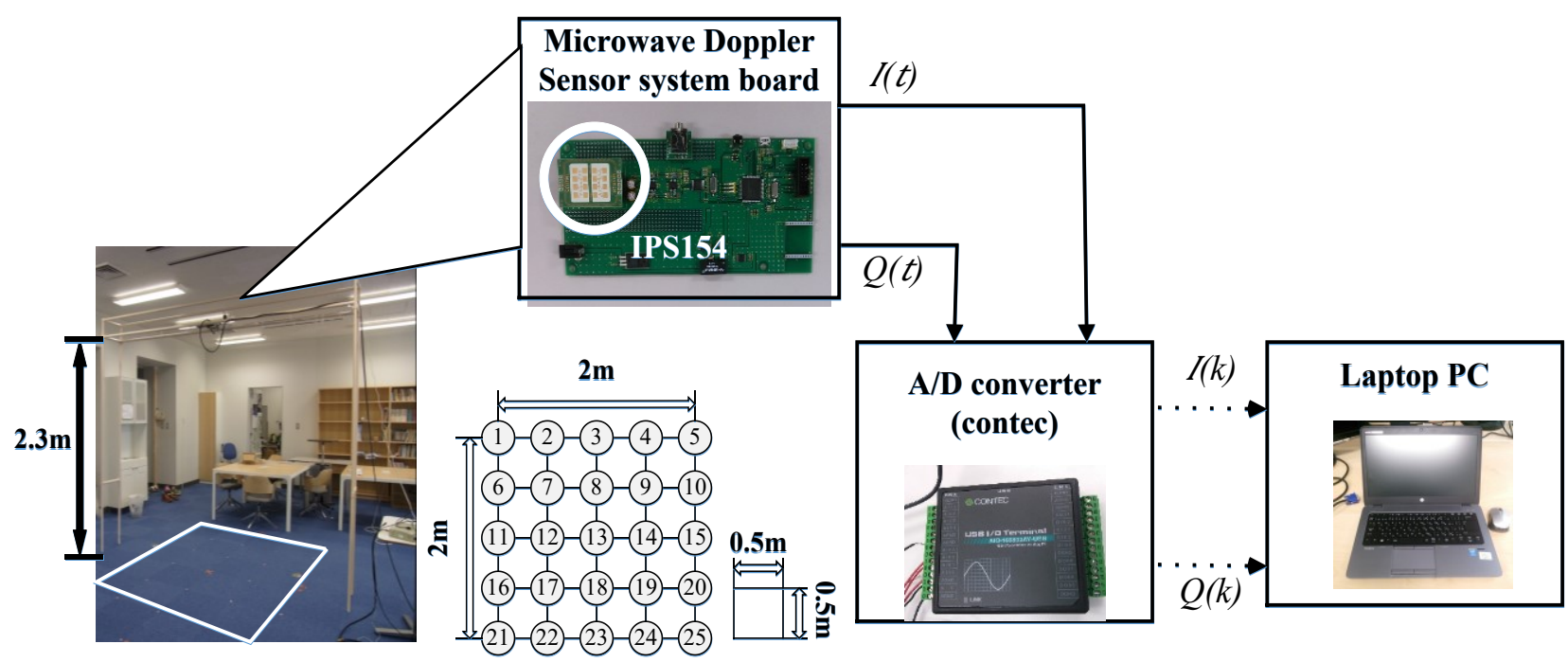

Fig. 2 Overview of our experimental system. Microwave Doppler sensor system board is set $2.3 \mathrm{~m}$ upside from the floor, simulating to set general room's ceiling. By A/D converter, two signals are converted from analog(continuous-time) to digital (discrete-time), for dealing with as csv file. 


\subsection{Method}

The data corresponding to the present class was obtained by measuring the subjects in our experiment area with two types of motion: a rest-state, where the subjects were quietly seated at each of the 25 positions. The distance from the sensor device critically affects the values of the feature vector used in the system. In total, 275 data points were obtained for the 11 data subjects. For the other considered motion, the moving-state, the subjects walked randomly in the experiment area. Within the 30 seconds of the experimental period, the subjects were asked to stand quiet for 10 seconds, then walk for 10 seconds, and stand quiet again for the remaining 10 seconds. In total, 550 data for the present state were obtained. The same number of absent data was collected, when no humans were present in the experiment area. The total number of absent data was therefore 550. The selection of a kernel for SVM is one of the key factors controlling system performance. Here, three kernel methods are used: linear, quadratic, and radial basis function (RBF). The accuracies obtained with each kernel are compared.

\subsection{Evaluation}

The performance of the proposed system is evaluated by its accuracy, precision, and recall, with a leave-one-subject-out approach. Given a test dataset whose true presence/absence labels are known, the classification results are grouped according to the four-element classification matrix shown in Table 1 . The true positive count (TP) is the number of cases where the system correctly classified a presence. The true negative count (TN) is the number of cases where the system correctly classified absence. The false positive count (FP) is the number of cases where the system erroneously classified presence (the subject was in fact absent). Finally, the false negative count $(\mathrm{FN})$ is the number of cases where the system erroneously classified absence. Both FP and FN are considered errors.

Using the prediction matrix entries, accuracy is then defined as follows:

$$
\text { Accuracy }=\frac{T P+T N}{T P+F P+F N+T N} .
$$

Table 1 Classification Matrix Summary

\begin{tabular}{|c|c|c|c|}
\hline \multicolumn{2}{|c|}{} & \multicolumn{2}{c|}{ Classified } \\
\cline { 3 - 4 } \multicolumn{2}{|c|}{ True } & Presence & Absence \\
\cline { 2 - 4 } & Absence & TP & FN \\
\hline \multirow{2}{*}{ FP } & TN \\
\hline
\end{tabular}

Table 2 Detailed Classification Matrix

\begin{tabular}{|c|c|c|c|}
\hline \multicolumn{2}{|c|}{} & \multicolumn{2}{|c|}{ Classified } \\
\cline { 3 - 4 } & Presence & Absence \\
\hline \multirow{4}{*}{ True } & Presence & $\begin{array}{c}\mathrm{TPR}= \\
\mathrm{TP} /(\mathrm{TP}+\mathrm{FN})\end{array}$ & $\begin{array}{c}\mathrm{FNR}= \\
\mathrm{FN} /(\mathrm{TP}+\mathrm{FN})\end{array}$ \\
\cline { 3 - 4 } & & $\mathrm{PPV}=$ & $\mathrm{FOR}=$ \\
& & $\mathrm{TP} /(\mathrm{TP}+\mathrm{FP})$ & $\mathrm{FN} /(\mathrm{FN}+\mathrm{TN})$ \\
\cline { 3 - 4 } & Absence & $\mathrm{FPR}=$ & $\mathrm{TNR}=$ \\
& & $\mathrm{FP} /(\mathrm{TP}+\mathrm{FP})$ & $\mathrm{TN} /(\mathrm{FN}+\mathrm{TN})$ \\
\cline { 3 - 4 } & & $\begin{array}{c}\mathrm{FDR}= \\
\mathrm{FP} /(\mathrm{TP}+\mathrm{FP})\end{array}$ & $\begin{array}{c}\mathrm{NPV}= \\
\mathrm{FN} /(\mathrm{FN}+\mathrm{TN})\end{array}$ \\
\hline
\end{tabular}

Additionally, eight sub-types of accuracy are obtained: TPR, FNR, FPR, TNR, PPV, FOR, FDR, and NPV, as defined in Table 2 .

\section{Experimental Results}

\subsection{Observed Data}

\subsubsection{Presence Data}

Fig. 3(a) shows a typical example of the data collected when a subject (ID1) was walking in the experiment area. The left chart is the time series for signal $I, I(k)$. In the period 10-20 seconds, while the subject was walking, the received voltage varies substantially. The right chart is the amplitude spectrum of signal $I, F_{I}(f)$. Complex respiration-induced spectral contents can be observed in the $0.2 \mathrm{~Hz}$ to $0.8 \mathrm{~Hz}$ frequency band, indicating that the respiratory period varied substantially. A similar complexity (and, therefore, variability) can be observed in the heartbeat-induced spectral contents, from $0.8 \mathrm{~Hz}$ to $1.5 \mathrm{~Hz}$.

Fig. 3(b) shows a typical example of the data observed when a subject (ID1) is located at position 13, whereas Fig.3(c) depicts the same subject (ID1), but this time located at position 1 . Notice that when a subject is present near the sensor device - position 13 - the time-series and its frequency spectrum show dynamics and complexity, but when a subject is located far from the sensor device- the case of position 1 - the frequency spectrum is mostly flat.

\subsubsection{Absence Data}

Fig. 3(d) shows the typical data observed when a subject is absent from the experiment area. Notice the low amplitudes of the time domain plot, and the flatness of the amplitude spectrum.

\subsection{Calculated Feature Vector}

Fig. 4 shows a scatter plot of all combinations of the calculated four-dimensional feature vector $\left(Y I_{R}, Y Q_{R}, Y I_{H}\right.$, and $\left.Y Q_{H}\right)$. Crosses represent presence data, and dots 
represent absence data. As shown, the presence and plots, enabling a good classification performance. absence data points are sufficiently separated in all scatter
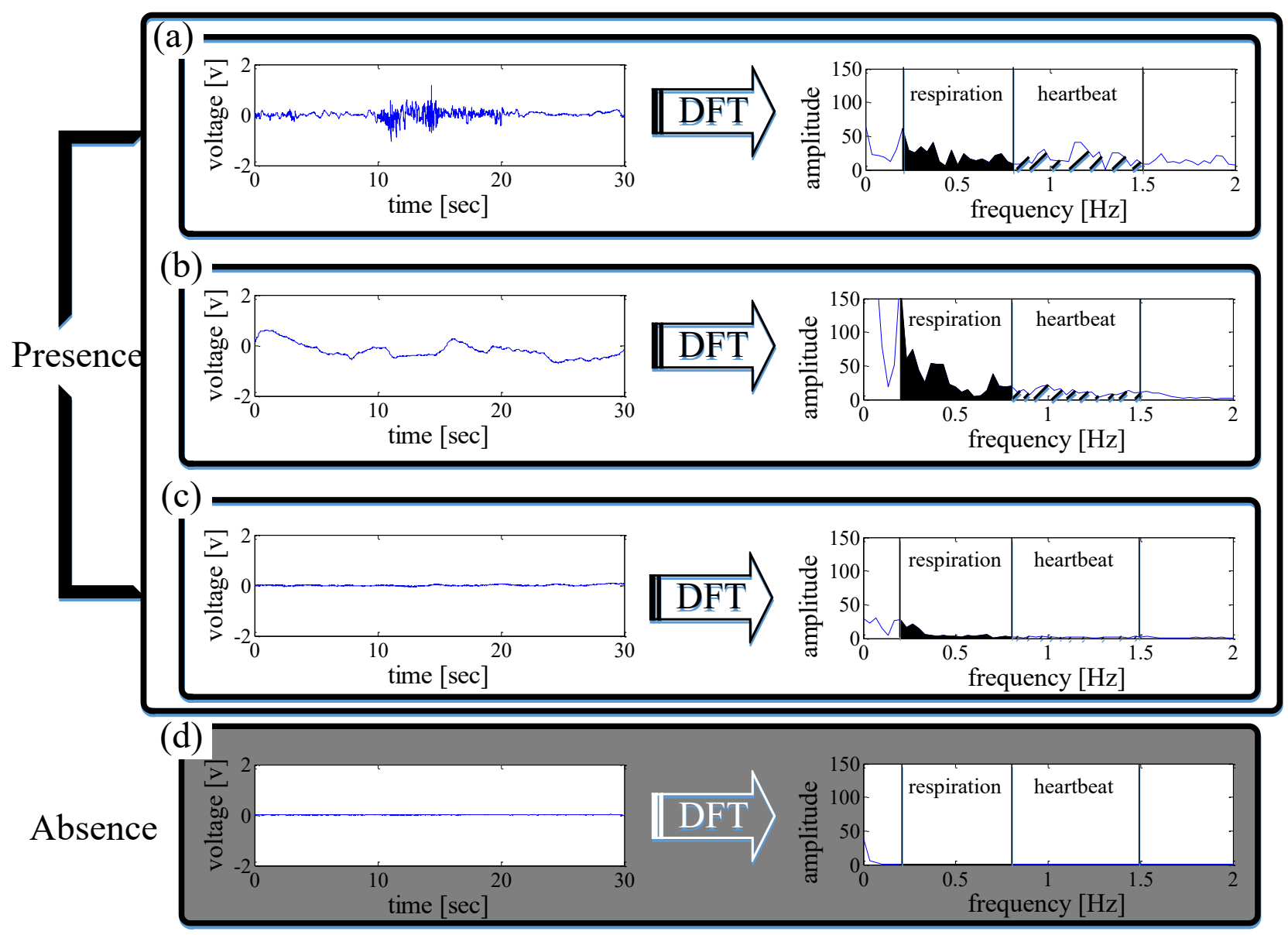

Fig. 3 one example of measured data and analysis. (a), (b), and (c) show presence data of signal I. (a) shows the data subject was walking from 10 seconds to 20 seconds. (b) and (c) show the data subject was seated position 13 and 1. (d) shows the absence data.
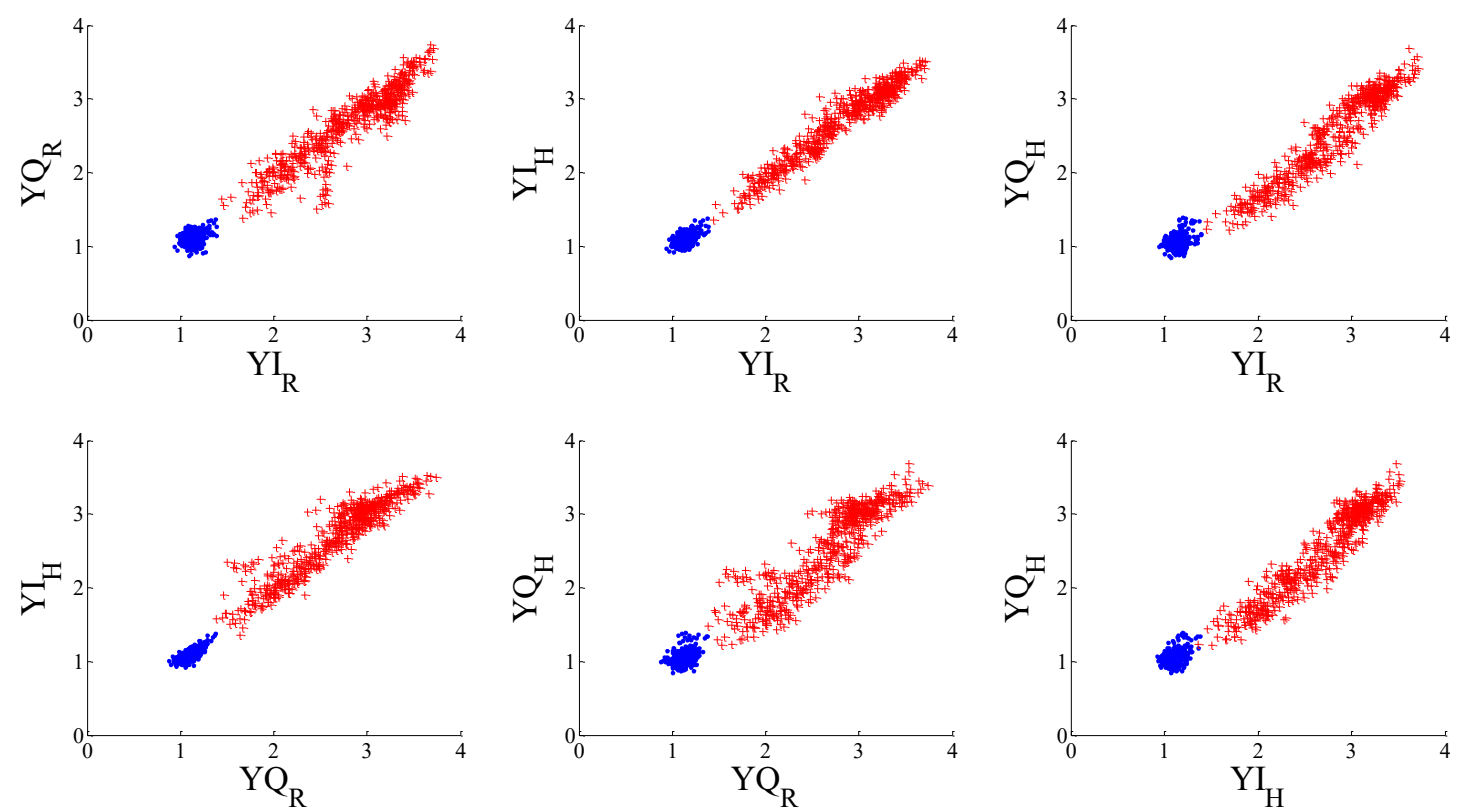

Fig.4 all pattern’s scatter chart of feature. Dots represent "absent" data, crosses represent "present" data. 


\subsection{Classification Results}

Tables 3, 4, 5, and 6 show the accuracies obtained with the proposed method, for each SVM kernel. As shown in Table 3, the linear kernel exhibited the worst accuracy (94.82\%). Using a quadratic kernel or an RBF kernel, the human's presence/absence was classified with an accuracy above $99.5 \%$. The RBF kernel had the best performance, with $99.73 \%$ accuracy.

It should also be noted that the absent state could be detected with $100 \%$ certainty by all kernels, as shown in Tables 4, 5, and 6 .

\section{Discussion}

Even though the linear kernel showed a slightly worse performance than the other two kernels, the experimental results show that the selection of the SVM kernel does not drastically affect the performance of the proposed system. This may be a result the non-linearity of the underlying distribution of features in the high-dimensional space. A precise comparison with previously existing related work is difficult, due to the uniqueness of the setup. However, the accuracy of the proposed method is higher than those previously reported ${ }^{(8,}$ 9) (i.e., $89.4 \%$ and $91.4 \%$, respectively). Furthermore, it should be noted that the proposed system can classify the subject's absence with

Table 3 Average classification results [\%]

\begin{tabular}{|c|c|c|c|}
\hline Kernel & Linear & Quadratic & RBF \\
\hline Accuracy & $94.82 \pm 2.59$ & $99.55 \pm 0.89$ & $99.73 \pm 0.62$ \\
\hline
\end{tabular}

Table 4 Average SVM classification accuracy with a linear kernel [\%]

\begin{tabular}{|c|c|c|c|}
\hline \multicolumn{2}{|c|}{} & \multicolumn{2}{|c|}{ Classified } \\
\cline { 3 - 4 } \multicolumn{2}{|c|}{} & Presence & Absence \\
\hline \multirow{3}{*}{ True } & Presence & $89.64 \pm 5.43$ & $10.36 \pm 5.43$ \\
\cline { 3 - 4 } & & $100.00 \pm 0.00$ & $91.89 \pm 4.51$ \\
\cline { 3 - 4 } & Absence & $0.00 \pm 0.00$ & $100.00 \pm 0.00$ \\
\cline { 3 - 4 } & & $0.00 \pm 0.00$ & $90.81 \pm 4.51$ \\
\hline
\end{tabular}

Table 5 Average SVM classification accuracy with quadratic kernel [\%]

\begin{tabular}{|c|c|c|c|}
\hline \multicolumn{2}{|c|}{} & \multicolumn{2}{|c|}{ Classified } \\
\cline { 3 - 4 } \multicolumn{2}{|c|}{ True } & Presence & Absence \\
\hline \multirow{3}{*}{} & & $99.09 \pm 1.87$ & $0.91 \pm 1.87$ \\
\cline { 3 - 4 } & Absence & $100.00 \pm 0.00$ & $0.87 \pm 1.77$ \\
\cline { 3 - 4 } & & $0.00 \pm 0.00$ & $100.00 \pm 0.00$ \\
\cline { 3 - 4 } & & $0.00 \pm 0.00$ & $99.13 \pm 1.77$ \\
\hline
\end{tabular}

$100 \%$ reliability. Therefore, the wandering of subjects to outside the home can be efficiently recognized.

From Fig. 3, one can observe that the spectral characteristics of the received signal when the subject is walking are different from the ones obtained when the subject is sitting quietly. Note also that the particular position of the subject will affect the feature vector. Therefore, a method to normalize these spectral characteristics is envisaged as a topic for future work.

\section{Conclusion}

We proposed a novel system to classify the presence or absence of a subject within a designated area using a microwave Doppler sensor. The proposed system uses the signals of respiration and heartbeat obtained from the sensor and then proceeds to classify the obtained data using an SVM. The system was evaluated using 11 subjects, and was shown to be capable of properly classifying the presence or absence of a human subject within an area of $2.0 \mathrm{~m} \times 2.0 \mathrm{~m}$ with a $99.73 \%$ accuracy. Wider ranges can be dealt with by using several such devices.

If one desires to wonder starting from a sitting state, he/she needs to stand up and walk to the door to get outside. Therefore, by individually classifying the walk and rest-states, the performance of the wandering detection systems is expected to still be amenable to further improvement.

Table 6 Average SVM classification accuracy with RBF kernel [\%]

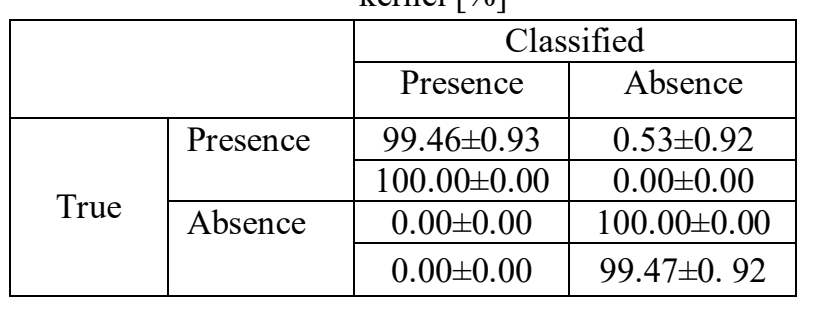

\section{Acknowledgment}

This work was supported by JSPS KAKENHI Grant Number 16K16392.

\section{References}

(1) United Nations, Department of Economic and Social Affairs : "World Population Ageing," 
(ST/ESA/SER.A/390), p. 2, 2015

(2) J. Heinik : 'Families' and professional caregivers' R. Laudau, G.K. Auslander, S. Werner, N. Shoval, and views of using advanced technology to track people with dementia," Psychogeriatrics, Vol. 1, No. 2, pp. 20-35, 2010

(3) N. M. Silverstein, G. Flaherty, and T. S. Tobin : "Dementia and Wandering Behavior: Concern for the Lost Elder," New York, NY, USA, Springer, pp. 28-51, 2006

(4) G. Cipriani, C. Lucetti, A. Nuti, and S. Danti : "Wandering and Dementia," Psychogeriatrics, Vol. 14, No. 2, pp. 135-142, 2014

(5) D. Okuya, M. Hiramoto, and K. Maeno : "Human Sensing Technique Using Microwave Doppler Radar Based on Higher-order Local Autocorrelation Features," IEICE Technical Report, Vol. 113, No. 28, pp. 13-18, 2013

(6) C.-Y. Ko, F.-Y. Leu, and I.-T. Lin : "A Wandering Path Tracking and Fall Detection System for People with Dementia," Broadband and Wireless Computing, Communication and Applications, pp. 306-311, 2014. DOI:10.1109/BWCCA.2014.127

(7) M. Sekine, K. Maeno, and T. Kamakura : "Human Detection Algorithm for Doppler Radar Using Prediction Error in Autoregressive Model," IEEE International Symposium on Instrumentation and Control Technology, 2012. DOI: 10.1109/ISICT.2012.6291657

(8) M. Sekine, K. Maeno, and M. Nozaki : "Activity and State Recognition without Body-Attached Sensor Using Microwave Doppler Sensor," IPSJ SIG Technical Report, Vol.2009-HCI-135, No. 10, pp. 1-8, 2009

(9) A. Solanas, E. Batista, F. Borras, A. M. Balleste, and C. Patsakis : "Wandering analysis with mobile phones: On the relation between randomness and wandering," International Conference on Pervasive and Embedded Computing and Communication Systems, pp. 168-173, 2015

(10)K. Shiba, T. Kaburagi, K. Ozaki, S. Nakamura, and Y. Kurihara : "Classification Presence/Absence system incorporating respirational signals by microwave Doppler sensor," Proc. SICE Annual Conference 2016, pp. 1310-1315, 2016 